\title{
Bifurcation of positive periodic solutions of first-order impulsive differential equations
}

\author{
Ruyun Ma*, Bianxia Yang and Zhenyan Wang
}

"Correspondence: mary@nwnu.edu.cn

Department of Mathematics, Northwest Normal University Lanzhou, 730070, P.R. China

\begin{abstract}
We give a global description of the branches of positive solutions of first-order impulsive boundary value problem:

$$
\begin{cases}u^{\prime}(t)+a(t) u(t)=\lambda f(t, u(t)), & t \in(0,1), t \neq t_{k}, k=1, \ldots, p, \\ u\left(t_{k}^{+}\right)=u\left(t_{k}^{-}\right)+\lambda l_{k}\left(u\left(t_{k}\right)\right), & k=1, \ldots, p, \quad u(0)=u(1),\end{cases}
$$

which is not necessarily linearizable. Where $\lambda>0$ is a parameter, $0<t_{1}<t_{2}<\cdots<t_{p}<1$ are given impulsive points. Our approach is based on the Krein-Rutman theorem, topological degree, and global bifurcation techniques.

MSC: 34B10; 34B15; 34K15; 34K10; 34C25; 92D25

Keywords: Krein-Rutman theorem; topological degree; bifurcation from interval; impulsive boundary value problem; existence and multiplicity
\end{abstract}

\section{Introduction}

Some evolution processes are distinguished by the circumstance that at certain instants their evolution is subjected to a rapid change, that is, a jump in their states. Mathematically, this leads to an impulsive dynamical system. Differential equations involving impulsive effects occur in many applications: physics, population dynamics, ecology, biological systems, biotechnology, industrial robotic, pharmacokinetics, optimal control, etc. Therefore, the study of this class of impulsive differential equations has gained prominence and it is a rapidly growing field. See [1-9] and the references therein.

Let us consider the equation

$$
u^{\prime}(t)+a(t) u(t)=\lambda f(t, u(t)), \quad t \in \mathbb{J}^{\prime},
$$

subjected to the impulsive boundary condition

$$
u\left(t_{k}^{+}\right)=u\left(t_{k}^{-}\right)+\lambda I_{k}\left(u\left(t_{k}\right)\right), \quad k=1, \ldots, p, \quad u(0)=u(1),
$$

where $\lambda>0$ is a real parameter, $\mathbb{J}^{\prime}=[0,1] \backslash\left\{t_{1}, \ldots, t_{p}\right\}, 0<t_{1}<t_{2}<\cdots<t_{p}<1$ are given impulsive points. We make the following assumptions:

(H1) $a \in C([0,1], \mathbb{R})$ is a 1-periodic function and $\int_{0}^{1} a(t) d t>0$;

2012 Ma et al.: licensee Springer. This is an Open Access article distributed under the terms of the Creative Commons Attribution License (http://creativecommons.org/licenses/by/2.0), which permits unrestricted use, distribution, and reproduction in any medium, provided the original work is properly cited. 
(H2) $I_{k} \in C([0, \infty),[0, \infty)), k=1, \ldots, p, I_{k}(u)>0$ for $u>0$, there exist positive constants $I_{k}^{(0)}, I_{k}^{(\infty)} \in(0, \infty)$ such that

$$
I_{k}^{(0)}=\lim _{u \rightarrow 0^{+}} \frac{I_{k}(u)}{u}, \quad I_{k}^{(\infty)}=\lim _{u \rightarrow+\infty} \frac{I_{k}(u)}{u} ;
$$

(H3) $f \in C\left(\mathbb{J}^{\prime} \times[0, \infty),[0, \infty)\right)$ is 1 -periodic function with respect to the first variable, and $f\left(t_{k}^{+}, u\right), f\left(t_{k}^{-}, u\right)$ exist, $f\left(t_{k}^{-}, u\right)=f\left(t_{k}, u\right)$. Moreover, there exist functions $a_{0}, a^{0}, b_{\infty}, b^{\infty} \in C([0,1],[0, \infty))$ with $a_{0}(t), a^{0}(t), b_{\infty}(t), b^{\infty}(t) \not \equiv 0$ in any subinterval of $[0,1]$ such that

$$
a_{0}(t) u-\xi_{1}(t, u) \leq f(t, u) \leq a^{0}(t) u+\xi_{2}(t, u),
$$

where $\xi_{i} \in C([0,1] \times[0, \infty))$ with $\xi_{i}(t, u)=o(|u|)$ as $|u| \rightarrow 0$ uniformly for $t \in[0,1]$ $(i=1,2)$, and

$$
b_{\infty}(t) u-\zeta_{1}(t, u) \leq f(t, u) \leq b^{\infty}(t) u+\zeta_{2}(t, u)
$$

where $\zeta_{i} \in C([0,1] \times[0, \infty))$ with $\zeta_{i}(t, u)=o(|u|)$ as $|u| \rightarrow \infty$ uniformly for $t \in[0,1](i=1,2)$;

(H4) $f(t, u)>0,(t, u) \in[0,1] \times(0, \infty)$;

(H5) there exists function $c \in C([0,1],[0, \infty))$ and $c(t) \not \equiv 0$ in any subinterval of $[0,1]$ such that

$$
f(t, u) \geq c(t) u, \quad(t, u) \in[0,1] \times[0, \infty) .
$$

Some special cases of (1.1), (1.2) have been investigated. For example, Nieto [3] considered the (1.1), (1.2) with $\lambda \equiv 1, a \equiv 0$. By using Schaeffer's theorem, some sufficient conditions for existence of solutions of the IBVP (1.1), (1.2) with $\lambda \equiv 1, a \equiv 0$ were obtained.

Li, Nieto, and Shen [4] studied the existence of at least one positive periodic solutions of (1.1), (1.2) with $\lambda \equiv 1, a \equiv m$ ( $m$ is a constant). By using Schaeffer's fixed-point theorem, they got the solvability under $f$ satisfied at most linear growth and $I_{k}$ is bounded or $f$ is bounded and $I_{k}$ satisfied at most linear growth.

Liu [7] studied the existence and multiplicity of (1.1), (1.2) with $\lambda \equiv 1$, by using the fixedpoint theorem in cones, and he proved the following:

Theorem A ([7, Theorem 3.1.1]) Let (H1) hold. Assume that $f(t, u) \geq 0, I_{k}(u) \geq 0, u \geq 0$, and

$$
\max _{t \in[0,1]}\left\{M \int_{0}^{1} G(t, s) d s+W \sum_{k=1}^{p} G\left(t, t_{k}\right)\right\}<1
$$

and

$$
\min _{t \in[0,1]}\left\{e^{-\int_{0}^{1}|a(s)| d s} v \int_{0}^{1} G(t, s) d s+e^{-\int_{0}^{1}|a(s)| d s} w \sum_{k=1}^{p} G\left(t, t_{k}\right)\right\}>1 .
$$


Then the problem (1.1), (1.2) with $\lambda \equiv 1$ has at least one positive solution where $G(t, s)$ will be defined in (2.2) and

$$
\begin{aligned}
& M:=\lim _{u \rightarrow+\infty} \sup _{t \in[0,1]} \frac{f(t, u)}{u}, \quad W_{k}:=\lim _{u \rightarrow+\infty} \frac{I_{k}(u)}{u}, \\
& v:=\lim _{u \rightarrow 0} \inf _{t \in[0,1]} \frac{f(t, u)}{u}, \quad w_{k}:=\lim _{u \rightarrow 0} \frac{I_{k}(u)}{u} .
\end{aligned}
$$

Theorem B ([7, Theorem 3.1.2]) Let (H1) hold. Assume that $f(t, u) \geq 0, I_{k}(u) \geq 0, u \geq 0$ and

$$
\min _{t \in[0,1]}\left\{m \int_{0}^{1} G(t, s) d s+W \sum_{k=1}^{p} G\left(t, t_{k}\right)\right\}>e^{\int_{0}^{1}|a(t)| d t},
$$

and

$$
\max _{t \in[0,1]}\left\{V \int_{0}^{1} G(t, s) d s+w \sum_{k=1}^{p} G\left(t, t_{k}\right)\right\}<1 .
$$

Then the problem (1.1), (1.2) with $\lambda \equiv 1$ has at least one positive solution where $W, w$ defined as (1.5) and

$$
m:=\lim _{u \rightarrow+\infty} \inf _{t \in[0,1]} \frac{f(t, u)}{u}, \quad V:=\lim _{u \rightarrow 0} \sup _{t \in[0,1]} \frac{f(t, u)}{u} .
$$

It is worth remarking that the $[3,4,7]$ only get the existence of solutions, and there is not any information of global structure of positive periodic solutions.

By using global bifurcation techniques, we obtain a complete description of the global structure of positive solutions for (1.1), (1.2) under weaker conditions. More precisely, our main result is the following theorem.

Theorem 1.1 Let (H1), (H2), and (H3) hold. Suppose $f(t, 0)=0, t \in[0,1], I_{k}(0)=0, k=$ $1, \ldots, p$. Then

(i) $\left[\lambda_{1}\left(b^{\infty}\right), \lambda_{1}\left(b_{\infty}\right)\right]$ is a bifurcation interval of positive solutions from infinity for (1.1), (1.2), and there exists no bifurcation interval of positive solutions from infinity which is disjoint with $\left[\lambda_{1}\left(b^{\infty}\right), \lambda_{1}\left(b_{\infty}\right)\right]$. More precisely, there exists a component $\Sigma_{\infty}$ of positive solutions of (1.1), (1.2) which meets $\left[\lambda_{1}\left(b^{\infty}\right), \lambda_{1}\left(b_{\infty}\right)\right] \times\{\infty\}$, where $\lambda_{1}\left(b^{\infty}\right), \lambda_{1}\left(b_{\infty}\right)$ will be defined in Section 2;

(ii) $\left[\tilde{\lambda}_{1}\left(a^{0}\right), \tilde{\lambda}_{1}\left(a_{0}\right)\right]$ is a bifurcation interval of positive solutions from the trivial solutions for (1.1), (1.2), and there exists no bifurcation interval of positive solutions from the trivial solutions which is disjoint with $\left[\tilde{\lambda}_{1}\left(a^{0}\right), \tilde{\lambda}_{1}\left(a_{0}\right)\right]$. More precisely, there exists a component $\Sigma_{0}$ of positive solutions of $(1.1),(1.2)$ which meets $\left[\tilde{\lambda}_{1}\left(a^{0}\right), \tilde{\lambda}_{1}\left(a_{0}\right)\right] \times\{0\}$, where $\tilde{\lambda}_{1}\left(a^{0}\right), \tilde{\lambda}_{1}\left(a_{0}\right)$ will be defined in Section 4;

(iii) If (H4) and (H5) also hold, then there is a number $\lambda^{*}>0$ such that problem (1.1), (1.2) admits no positive solution with $\lambda>\lambda^{*}$. In this case, $\Sigma_{\infty}=\Sigma_{0}$.

Remark 1.1 There is no paper except [9] studying impulsive differential equations using bifurcation ideas. However, in [9], they only dealt with the case that $f_{0}, f_{\infty} \in(0, \infty)$, i.e. $f_{0}$, 
$f_{\infty}$ do exist. Where

$$
\begin{aligned}
& f_{0}:=\lim _{|u| \rightarrow 0} \frac{f(t, u)}{u} \text { and } \\
& f_{\infty}:=\lim _{|u| \rightarrow \infty} \frac{f(t, u)}{u} \text { both uniformly with respect to } t \in[0,1] .
\end{aligned}
$$

From (H3), it is easy to see that the $f_{0}, f_{\infty}$ may be not exist, the method used in [9] is not helpful any more in this case.

Remark 1.2 From (iii) of Theorem 1.1, we know that $\Sigma_{0}, \Sigma_{\infty}$ are involved in $\left[0, \lambda^{*}\right] \times$ $P C[0,1]$. Moreover, $\left[\lambda_{1}\left(b^{\infty}\right), \lambda_{1}\left(b_{\infty}\right)\right]$ is a unique bifurcation interval of positive solutions from infinity for (1.1), (1.2), and $\left[\tilde{\lambda}_{1}\left(a^{0}\right), \tilde{\lambda}_{1}\left(a_{0}\right)\right]$ is a unique bifurcation interval of positive solutions from the trivial solutions for (1.1), (1.2). Therefore, $\Sigma_{0}$ must be intersected with $\left[\lambda_{1}\left(b^{\infty}\right), \lambda_{1}\left(b_{\infty}\right)\right] \times\{\infty\}$.

Remark 1.3 Obviously, (H3) is more general than (1.5), (1.8). Moreover, if we let $a_{0}(t):=v$, $b^{\infty}(t):=M$, under conditions (1.3), (1.4), we get $\lambda_{1}\left(b^{\infty}\right)>1, \tilde{\lambda}_{1}\left(a_{0}\right)<1$, respectively. Hence, $\Sigma_{0}$ cross the hyperplane $\{1\} \times P C[0,1]$. Therefore, Theorem 3.1 .1 of [7] is the corollary of Theorems 1.1 even in the special case.

Remark 1.4 Similar, if we let $a^{0}(t):=V, b_{\infty}(t):=m$, only under condition (1.6), we can obtain $\lambda_{1}\left(b_{\infty}\right)<1$. From Proposition 3.1, we will know that $\Sigma_{\infty}$ is unbounded in $\lambda$ direction, so, $\Sigma_{\infty}$ cross the hyperplane $\{1\} \times P C[0,1]$. Therefore, Theorem 3.1.2 of [7] is the corollary of Theorems 1.1 even in the special case and weaker condition.

Remark 1.5 There are many papers which get the positive solutions using bifurcation from the interval. For example, see $[10,11]$. However, in those papers, the linear operator corresponding problem is self-adjoint. It is easy to see that the linear operator corresponding $(1.1),(1.2)$ is not self-adjoint. So, the method used in $[9,10]$ is not helpful in this case.

Remark 1.6 Condition (H3) means that $f$ is not necessarily linearizable near 0 and infinity. So, we will apply the following global bifurcation theorems for mappings which are not necessarily smooth to get a global description of the branches of positive solutions of (1.1), (1.2), and then, we obtain the existence and multiplicity of positive solutions of (1.1), (1.2).

Theorem C (K. Schmitt, R. C. Thompson [12]) Let V be a real reflexive Banach space. Let $F: \mathbb{R} \times V \rightarrow V$ be completely continuous such that $F(\lambda, 0)=0, \forall \lambda \in \mathbb{R}$. Let $a, b \in \mathbb{R}(a<b)$ be such that $u=0$ is an isolated solution of the equation

$$
u-F(\lambda, u)=0, \quad u \in V,
$$

for $\lambda=a$ and $\lambda=b$, where $(a, 0),(b, 0)$ are not bifurcation points of (1.9). Furthermore, assume that

$$
\operatorname{deg}\left(I-F(a, \cdot), B_{r}(0), 0\right) \neq \operatorname{deg}\left(I-F(b, \cdot), B_{r}(0), 0\right),
$$


where $B_{r}(0)$ is an isolating neighborhood of the trivial solution. Let

$$
\ell=\overline{\{(\lambda, u):(\lambda, u) \text { is a solution of (1.9) with } u \neq 0\}} \cup([a, b] \times\{0\}) .
$$

Then there exists a connected component $\mathcal{C}$ of $\ell$ containing $[a, b] \times\{0\}$ in $\mathbb{R} \times V$, and either

(i) $\mathcal{C}$ is unbounded in $\mathbb{R} \times V$, or

(ii) $\mathcal{C} \cap[(\mathbb{R} \backslash[a, b]) \times\{0\}] \neq \emptyset$.

Theorem D (K. Schmitt [13]) Let $V$ be a real reflexive Banach space. Let $F: \mathbb{R} \times V \rightarrow V$ be completely continuous, and let $a, b \in \mathbb{R}(a<b)$ be such that the solution of (1.9) are, $a$ priori, bounded in $V$ for $\lambda=a$ and $\lambda=b$, i.e., there exists an $R>0$ such that

$$
F(a, u) \neq u \neq F(b, u)
$$

for all $u$ with $\|u\| \geq R$. Furthermore, assume that

$$
\operatorname{deg}\left(I-F(a, \cdot), B_{R}(0), 0\right) \neq \operatorname{deg}\left(I-F(b, \cdot), B_{R}(0), 0\right),
$$

for $R>0$ large. Then there exists a closed connected set $\mathcal{C}$ of solutions of (1.9) that is unbounded in $[a, b] \times V$, and either

(i) $\mathcal{C}$ is unbounded in $\lambda$ direction, or

(ii) there exist an interval $[c, d]$ such that $(a, b) \cap(c, d)=\emptyset$, and $\mathcal{C}$ bifurcates from infinity in $[c, d] \times V$.

The rest of the paper is organized as follows: In Section 2, we state some notations and preliminary results. Sections 3 and 4 are devoted to study the bifurcation from infinity and from the trivial solution for a nonlinear problem which are not necessarily linearizable, respectively. Finally, in Section 5, we consider the intertwining of the branches bifurcating from infinity and from the trivial solution.

\section{Preliminaries}

Let

$$
P C[0,1]=\left\{\begin{array}{l}
u:[0,1] \rightarrow \mathbb{R}, u(t) \text { is continuous at } t \neq t_{k}, \\
\text { left continuous at } t=t_{k}, \text { and the right limit } u\left(t_{k}^{+}\right) \text {exists } \\
\text { for } k=1,2,3, \ldots
\end{array}\right\}
$$

Then $P C[0,1]$ is a Banach space with the norm $\|u\|=\sup _{t \in[0,1]}|u(t)|$.

By a positive solution of the problem (1.1), (1.2), we mean a pair $(\lambda, u)$, where $\lambda>0$ and $u$ is a solution of (1.1), (1.2) with $u>0$. Let $\Sigma \subset \mathbb{R}^{+} \times P C[0,1]$ be the closure of the set of positive solutions of (1.1), (1.2), where $\mathbb{R}_{+}:=[0, \infty)$.

Lemma 2.1 ([14, Theorem 6.26]) The spectrum $\sigma(T)$ of compact linear operator $T$ has following properties:

(i) $\sigma(T)$ is a countable set with no accumulation point which is different from zero;

(ii) each nonzero $\lambda \in \sigma(T)$ is an eigenvalue of $T$ with finite multiplicity, and $\bar{\lambda}$ is an eigenvalue of $T^{*}$ with the same multiplicity, where $\bar{\lambda}$ denote the conjugate of $\lambda, T^{*}$ denote the conjugate operator of $T$. 
Let $H:=L^{2}(0,1)$, with inner product $\langle\cdot, \cdot\rangle$ and norm $\|\cdot\|_{L^{2}}$.

Let $Z(\cdot) \in C([0,1],[0, \infty))$ and $Z(\cdot) \not \equiv 0$ in any subinterval of $[0,1]$. Further define the linear operator $L_{Z}: P C[0,1] \rightarrow P C[0,1]$,

$$
L_{Z} u=\int_{0}^{1} G(t, s) Z(s) u(s) d s+\sum_{k=1}^{p} G\left(t, t_{k}\right) I_{k}^{(\infty)} \cdot u\left(t_{k}\right)
$$

where $I_{k}^{(\infty)}$ as defined in (H2), $G(t, s)$ is the Green's function of

$$
\left\{\begin{array}{l}
u^{\prime}(t)+a(t) u(t)=0, \quad t \in(0,1) \\
u(0)=u(1)
\end{array}\right.
$$

and

$$
G(t, s)= \begin{cases}\frac{e^{-[A(t)-A(s)]}}{1-e^{-A(1)}}, & 0 \leq s \leq t \leq 1, \\ \frac{e^{-[A(1)+A(t)-A(s)]}}{1-e^{-A(1)}}, & 0 \leq t<s \leq 1,\end{cases}
$$

where $A(t)=\int_{0}^{t} a(s) d s$, it is easy to see that $(\mathrm{H} 1)$ implies that $G(t, s)>0$.

By virtue of Krein-Rutman theorems (see [15]), we have the following lemma.

Lemma 2.2 Suppose that (H1) holds, then for the operator $L_{Z}$ defined by (2.1), has a unique characteristic value $\lambda_{1}(Z)$, which is positive, real, simple, and the corresponding eigenfunction $\varphi(t)$ is of one sign, i.e., we have $\varphi(t)=\lambda_{1}(Z) L_{Z} \varphi(t)$.

Proof It is a direct consequence of the Krein-Rutman theorem [15, Theorem 19.3].

Remark 2.1 Since $\lambda_{1}(Z)$ is real number, so from Lemma 2.1, $\lambda_{1}(Z)$ is also the characteristic value of $L_{Z}^{*}$, let $\varphi_{1}^{*}$ denote the nonnegative eigenfunction of $L_{Z}^{*}$ corresponding to $\lambda_{1}(Z)$, where $L_{Z}^{*}$ denote the conjugate operator of $L_{Z}$. Therefore, we have

$$
\varphi_{1}^{*}(t)=\lambda_{1}(Z) L_{Z}^{*} \varphi_{1}^{*}(t), \quad t \in[0,1] .
$$

We extend the function $f$ to function $\bar{f}$, defined on $[0,1] \times \mathbb{R}$ by

$$
\bar{f}(t, u)= \begin{cases}f(t, u), & (t, u) \in[0,1] \times[0, \infty), \\ f(t, 0), & (t, u) \in[0,1] \times(-\infty, 0) .\end{cases}
$$

Then $\bar{f}(t, u) \geq 0$ on $[0,1] \times \mathbb{R}$.

For $\lambda \geq 0$, the problem

$$
\left\{\begin{array}{l}
u^{\prime}(t)+a(t) u(t)=\lambda \bar{f}(t, u(t)), \quad t \in \mathbb{J}^{\prime}, \\
u\left(t_{k}^{+}\right)=u\left(t_{k}^{-}\right)+\lambda I_{k}\left(u\left(t_{k}\right)\right), \quad k=1, \ldots, p, \quad u(0)=u(1)
\end{array}\right.
$$

is equivalent to the operator equation $A_{\lambda}: P C[0,1] \rightarrow P C[0,1]$.

$$
\left(A_{\lambda} u\right)(t)=\lambda \int_{0}^{1} G(t, s) \bar{f}(s, u(s)) d s+\lambda \sum_{k=1}^{p} G\left(t, t_{k}\right) I_{k}\left(u\left(t_{k}\right)\right) .
$$


Remark 2.2 For $\lambda>0$, if $u$ is a nontrivial solution of (2.3), from the positivity of $G(t, s)$ and $\bar{f}$, we have that $u(\cdot)>0$ on $[0,1]$, so $u$ is a nontrivial solution of (1.1), (1.2). Therefore, the closure of the set of nontrivial solutions $(\lambda, u)$ of $(2.3)$ in $\mathbb{R}^{+} \times P C[0,1]$ is exactly $\Sigma$.

The problem (2.3) is now equivalent to the operator equation

$$
u=A_{\lambda}(u), \quad u \in P C[0,1]
$$

In the following, we shall apply the Leray-Schauder degree theory, mainly to the mapping $\Phi_{\lambda}: P C[0,1] \rightarrow P C[0,1]$,

$$
\Phi_{\lambda}(u)=u-A_{\lambda}(u) .
$$

For $R>0$, let $B_{R}=\{u \in P C[0,1]:\|u\|<R\}$, let $\operatorname{deg}\left(\Phi_{\lambda}, B_{R}, 0\right)$ denote the degree of $\Phi_{\lambda}$ on $B_{R}$ with respect to 0 .

\section{Bifurcation from infinity}

In this section, we are devoted to study the bifurcation from infinity.

Lemma 3.1 Let $\Lambda \subset \mathbb{R}^{+}$be a compact interval with $\left[\lambda_{1}\left(b^{\infty}\right), \lambda_{1}\left(b_{\infty}\right)\right] \cap \Lambda=\emptyset$. Then there exists a number $R_{1}>0$ such that

$$
\Phi_{\lambda}(u) \neq 0, \quad \forall \lambda \in \Lambda, \forall u \in P C[0,1]:\|u\| \geq R_{1} .
$$

Proof Suppose on the contrary that there exists $\left\{\left(\mu_{n}, u_{n}\right)\right\} \subset \Lambda \times P C[0,1]$ with $\left\|u_{n}\right\| \rightarrow \infty$ $(n \rightarrow \infty)$, such that $\Phi_{\mu_{n}}\left(u_{n}\right)=0$. We may assume $\mu_{n} \rightarrow \bar{\mu} \in \Lambda$. By Remark 2.2, $u_{n}>0$ in $[0,1]$. Set $v_{n}=\left\|u_{n}\right\|^{-1} u_{n}$. Then

$$
v_{n}=\frac{A_{\mu_{n}}\left(u_{n}\right)}{\left\|u_{n}\right\|} .
$$

From (H2), (H3), we know that $\left\|u_{n}\right\|^{-1} A_{\mu_{n}}\left(u_{n}\right)$ is bounded in $P C[0,1]$, so $\left\{v_{n}\right\}$ is a relatively compact set in $P C[0,1]$ since $A_{\mu_{n}}: P C[0,1] \rightarrow P C^{1}[0,1]$ is bounded and continuous and $P C^{1}[0,1] \hookrightarrow \hookrightarrow P C[0,1]$. Suppose $v_{n} \rightarrow \bar{v}$ in $P C[0,1]$. Then $\|\bar{v}\|=1$ and $\bar{v} \geq 0$ in $[0,1]$.

Now, from condition $(\mathrm{H} 2)$, we know that there exist $\rho_{k} \in C([0, \infty),[0, \infty))$, such that

$$
I_{k}(u)=I_{k}^{(\infty)} u+\rho_{k}^{(\infty)}(u) \quad \text { and } \quad \lim _{|u| \rightarrow \infty} \frac{\rho_{k}^{\infty}(u)}{u}=0 .
$$

From $(\mathrm{H} 3)$, we have that

$$
b_{\infty}(t) u_{n}-\xi_{1}\left(t, u_{n}\right) \leq f\left(t, u_{n}\right) \leq b^{\infty}(t) u_{n}+\xi_{2}\left(t, u_{n}\right) .
$$

So,

$$
\begin{aligned}
u_{n} \leq & \mu_{n} \int_{0}^{1} G(t, s) b^{\infty}(s) u_{n}(s) d s+\mu_{n} \sum_{k=1}^{p} G\left(t, t_{k}\right) I_{k}^{(\infty)} \cdot u_{n}\left(t_{k}\right) \\
& +\mu_{n} \int_{0}^{1} G(t, s) \xi_{2}\left(s, u_{n}(s)\right) d s+\mu_{n} \sum_{k=1}^{p} G\left(t, t_{k}\right) \rho_{k}^{\infty}\left(u_{n}\left(t_{k}\right)\right),
\end{aligned}
$$


and

$$
\begin{aligned}
& \mu_{n} \int_{0}^{1} G(t, s) b_{\infty}(s) u_{n}(s) d s+\mu_{n} \sum_{k=1}^{p} G\left(t, t_{k}\right) I_{k}^{(\infty)} \cdot u_{n}\left(t_{k}\right) \\
& \quad-\mu_{n} \int_{0}^{1} G(t, s) \xi_{1}\left(s, u_{n}(s)\right) d s+\mu_{n} \sum_{k=1}^{p} G\left(t, t_{k}\right) \rho_{k}^{\infty}\left(u_{n}\left(t_{k}\right)\right) \leq u_{n}
\end{aligned}
$$

accordingly, we have

$$
\begin{aligned}
v_{n} \leq & \mu_{n} \int_{0}^{1} G(t, s) b^{\infty}(s) v_{n}(s) d s+\mu_{n} \sum_{k=1}^{p} G\left(t, t_{k}\right) I_{k}^{(\infty)} \cdot v_{n}\left(t_{k}\right) \\
& +\mu_{n} \int_{0}^{1} G(t, s) \frac{\xi_{2}\left(s, u_{n}(s)\right)}{u_{n}(s)} v_{n}(s) d s+\mu_{n} \sum_{k=1}^{p} G\left(t, t_{k}\right) \frac{\rho_{k}^{\infty}\left(u_{n}\left(t_{k}\right)\right)}{\left\|u_{n}\right\|}
\end{aligned}
$$

and

$$
\begin{aligned}
v_{n} \geq & \mu_{n} \int_{0}^{1} G(t, s) b_{\infty}(s) v_{n}(s) d s+\mu_{n} \sum_{k=1}^{p} G\left(t, t_{k}\right) I_{k}^{(\infty)} \cdot v_{n}\left(t_{k}\right) \\
& \quad-m u_{n} \int_{0}^{1} G(t, s) \frac{\xi_{1}\left(s, u_{n}(s)\right)}{u_{n}(s)} v_{n}(s) d s+\mu_{n} \sum_{k=1}^{p} G\left(t, t_{k}\right) \frac{\rho_{k}^{\infty}\left(u_{n}\left(t_{k}\right)\right)}{\left\|u_{n}\right\|} .
\end{aligned}
$$

Let $\varphi_{*}^{\infty}$ and $\varphi_{\infty}^{*}$ denote the nonnegative eigenfunctions of $L_{b}^{*}, L_{b_{\infty}}^{*}$ corresponding to $\lambda_{1}\left(b^{\infty}\right)$, and $\lambda_{1}\left(b_{\infty}\right)$, respectively. Then we have from the (3.1) that

$$
\left\langle v_{n}, \varphi_{*}^{\infty}\right\rangle \leq \mu_{n}\left\langle L_{b} \infty v_{n}, \varphi_{*}^{\infty}\right\rangle
$$

Letting $n \rightarrow \infty$, we have

$$
\left\langle\bar{v}, \varphi_{*}^{\infty}\right\rangle \leq \bar{\mu}\left\langle L_{b^{\infty}} \bar{v}, \varphi_{*}^{\infty}\right\rangle
$$

we obtain that

$$
\begin{aligned}
\left\langle\bar{v}, \varphi_{*}^{\infty}\right\rangle & \leq \bar{\mu}\left\langle L_{b^{\infty}} \bar{v}, \varphi_{*}^{\infty}\right\rangle=\bar{\mu}\left\langle\bar{v}, L_{b^{\infty}}^{*} \varphi_{*}^{\infty}\right\rangle \\
& =\bar{\mu}\left\langle\bar{v}, \frac{1}{\lambda_{1}\left(b^{\infty}\right)} \varphi_{*}^{\infty}\right\rangle=\bar{\mu} \frac{1}{\lambda_{1}\left(b^{\infty}\right)}\left\langle\bar{v}, \varphi_{*}^{\infty}\right\rangle,
\end{aligned}
$$

and consequently

$$
\bar{\mu} \geq \lambda_{1}\left(b^{\infty}\right) .
$$

Similarly, we deduce from (3.2) that

$$
\bar{\mu} \leq \lambda_{1}\left(b_{\infty}\right) .
$$

Thus, $\lambda_{1}\left(b^{\infty}\right) \leq \bar{\mu} \leq \lambda_{1}\left(b_{\infty}\right)$. This contradicts $\bar{\mu} \in \Lambda$. 
Corollary 3.1 For $\mu \in\left(0, \lambda_{1}\left(b^{\infty}\right)\right)$ and $R \geq R_{1}$. Then $\operatorname{deg}\left(\phi_{\mu}, B_{R}, 0\right)=1$.

Proof Lemma 3.1, applied to the interval $\Lambda=[0, \mu]$, guarantees the existence of $R_{1}>0$ such that for $R \geq R_{1}$,

$$
u-\tau A_{\mu}(u) \neq 0, \quad u \in P C[0,1]:\|u\| \geq R, \tau \in[0,1]
$$

Hence, for any $R \geq R_{1}$,

$$
\operatorname{deg}\left(\phi_{\mu}, B_{R}, 0\right)=\operatorname{deg}\left(I, B_{R}, 0\right)=1,
$$

which implies the assertion.

On the other hand, we have

Lemma 3.2 Suppose $\lambda>\lambda_{1}\left(b_{\infty}\right)$. Then there exists $R_{2}>0$ with the property that $\forall u \in$ $P C[0,1]$ with $\|u\| \geq R_{2}, \forall \tau \geq 0$,

$$
\Phi_{\lambda}(u) \neq \tau \varphi_{\infty}
$$

where $\varphi_{\infty}$ is the nonnegative eigenfunction of $L_{b_{\infty}}$ corresponding to $\lambda_{1}\left(b_{\infty}\right)$.

Proof Let us assume that for some sequence $\left\{u_{n}\right\}$ in $P C[0,1]$ with $\left\|u_{n}\right\| \rightarrow \infty$ and numbers $\tau_{n} \geq 0$, such that $\Phi_{\lambda}\left(u_{n}\right)=\tau_{n} \varphi_{\infty}$. Then

$$
u_{n}=A_{\lambda}\left(u_{n}\right)+\tau_{n} \varphi_{\infty}
$$

and we conclude from Remark 2.2 that $u_{n}>0$ in $[0,1]$. So we have

$$
\left\langle u_{n}, \varphi_{\infty}^{*}\right\rangle=\left\langle A_{\lambda}\left(u_{n}\right)+\tau_{n} \varphi_{\infty}, \varphi_{\infty}^{*}\right\rangle=\left\langle A_{\lambda}\left(u_{n}\right), \varphi_{\infty}^{*}\right\rangle+\tau_{n}\left\langle\varphi_{\infty}, \varphi_{\infty}^{*}\right\rangle
$$

Choose $\sigma>0$ such that

$$
\sigma<\frac{\lambda-\lambda_{1}\left(b_{\infty}\right)}{\lambda}
$$

By (H3), there exists $M_{0}>0$, such that

$$
f(t, u) \geq(1-\sigma) b_{\infty}(t) u, \quad \forall u>M_{0}, t \in[0,1]
$$

From $\left\|u_{n}\right\| \rightarrow \infty$, then exists $N^{*}>0$, such that

$$
u_{n}>M_{0}, \quad t \in[0,1], \forall n \geq N^{*}
$$

and consequently

$$
f\left(t, u_{n}\right) \geq(1-\sigma) b_{\infty}(t) u_{n}, \quad \forall n \geq N^{*}, t \in[0,1]
$$


Let $v_{n}=\frac{u_{n}}{\left\|u_{n}\right\|}$, applying (3.4), it follows that

$$
\begin{aligned}
\left\langle v_{n}, \varphi_{\infty}^{*}\right\rangle & \geq\left\langle\frac{A_{\lambda}\left(u_{n}\right)}{\left\|u_{n}\right\|}, \varphi_{\infty}^{*}\right\rangle \geq \lambda(1-\sigma)\left\langle L_{b_{\infty}} v_{n}, \varphi_{\infty}^{*}\right\rangle \\
& =\lambda(1-\sigma)\left\langle v_{n}, L_{b_{\infty}}^{*} \varphi_{\infty}^{*}\right\rangle=\lambda(1-\sigma)\left\langle v_{n}, \frac{1}{\lambda_{1}\left(b_{\infty}\right)} \varphi_{\infty}^{*}\right\rangle .
\end{aligned}
$$

Thus,

$$
\lambda_{1}\left(b_{\infty}\right) \geq \lambda(1-\sigma)
$$

this contradicts (3.3).

Corollary 3.2 For $\lambda>\lambda_{1}\left(b_{\infty}\right)$ and $R \geq R_{2}, \operatorname{deg}\left(\phi_{\lambda}, B_{R}, 0\right)=0$.

Proof By Lemma 3.2, there exists $R_{2}>0$ such that

$$
\Phi_{\lambda}(u) \neq \tau \varphi_{\infty}, \quad u \in P C[0,1]:\|u\| \geq R_{2}, \tau \in[0,1] .
$$

Then

$$
\operatorname{deg}\left(\Phi_{\lambda}, B_{R}, 0\right)=\operatorname{deg}\left(\Phi_{\lambda}-\varphi_{\infty}, B_{R}, 0\right)=0
$$

for all $R \geq R_{2}$. The assertion follows.

We are now ready to prove

Proposition $3.1\left[\lambda_{1}\left(b^{\infty}\right), \lambda_{1}\left(b_{\infty}\right)\right]$ is a bifurcation interval of positive solutions from infinity for the problem (2.4). There exists an unbounded component $\Sigma_{\infty}$ of positive solutions of (2.4) which meets $\left[\lambda_{1}\left(b^{\infty}\right), \lambda_{1}\left(b_{\infty}\right)\right] \times\{\infty\}$, and is unbounded in $\lambda$ direction. Moreover, there exists no bifurcation interval of positive solutions from infinity which is disjointed with $\left[\lambda_{1}\left(b^{\infty}\right), \lambda_{1}\left(b_{\infty}\right)\right]$.

Proof For fixed $n \in \mathbb{N}$ with $\lambda_{1}\left(b^{\infty}\right)-\frac{1}{n}>0$, let us take that $a_{n}=\lambda_{1}\left(b^{\infty}\right)-\frac{1}{n}, b_{n}=\lambda_{1}\left(b_{\infty}\right)+\frac{1}{n}$ and $\hat{R}=\max \left\{R_{1}, R_{2}\right\}$. It is easy to check that for $R>\hat{R}$, all of the conditions of Theorem $\mathrm{D}$ are satisfied. So, there exists a closed connected set $\mathcal{C}_{n}$ of solutions of (2.4) that is unbounded in $\left[a_{n}, b_{n}\right] \times P C[0,1]$, and either

(i) $\mathcal{C}_{n}$ is unbounded in $\lambda$ direction, or else

(ii) $\exists[c, d]$ such that $\left(a_{n}, b_{n}\right) \cap(c, d)=\emptyset$ and $\mathcal{C}_{n}$ bifurcates from infinity in $[c, d] \times P C[0,1]$.

By Lemma 3.1, the case (ii) cannot occur. Thus, $\mathcal{C}_{n}$ bifurcates from infinity in $\left[a_{n}, b_{n}\right] \times$ $P C[0,1]$ and is unbounded in $\lambda$ direction. Furthermore, we have from Lemma 3.1 that for any closed interval $I \subset\left[a_{n}, b_{n}\right] \backslash\left[\lambda_{1}\left(b^{\infty}\right), \lambda_{1}\left(b_{\infty}\right)\right]$, the set $\{u \in P C[0,1] \mid(\lambda, u) \in \Sigma, \lambda \in I\}$ is bounded in $P C[0,1]$. So, $\mathcal{C}_{n}$ must be bifurcated from infinity in $\left[\lambda_{1}\left(b^{\infty}\right), \lambda_{1}\left(b_{\infty}\right)\right] \times P C[0,1]$ and is unbounded in $\lambda$ direction.

Assertion (i) of Theorem 1.1 follows directly. 


\section{Bifurcation from the trivial solutions}

In this section, we shall study the bifurcation from the trivial solution for a nonlinear problem which is not necessarily linearizable near 0 and infinity.

As in Section 2 , let $Z(\cdot) \in C([0,1],[0, \infty))$ and $Z(\cdot) \not \equiv 0$ in any subinterval of $[0,1]$. Further define the linear operator $\tilde{L}_{Z}: P C[0,1] \rightarrow P C[0,1]$,

$$
\tilde{L}_{Z} u=\int_{0}^{1} G(t, s) Z(s) u(s) d s+\sum_{k=1}^{p} G\left(t, t_{k}\right) I_{k}^{(0)} \cdot u\left(t_{k}\right),
$$

where $I_{k}^{(0)}$ is defined in (H2), $G(t, s)$ is defined in (2.2).

Similar as Lemma 2.2, we have the following lemma.

Lemma 4.1 Suppose that (H1) holds, then the operator $\tilde{L}_{Z}$ has a unique characteristic value $\tilde{\lambda}_{1}(Z)$, which is positive, real, simple, and the corresponding eigenfunction $\tilde{\varphi}(t)$ is of one sign, i.e., we have $\tilde{\varphi}_{1}(t)=\tilde{\lambda}_{1}(Z) \tilde{L}_{Z} \tilde{\varphi}_{1}(t)$.

Remark 4.1 Since $\tilde{\lambda}_{1}(Z)$ is real number, so from Lemma $2.1, \tilde{\lambda}_{1}(Z)$ is also the characteristic value of $\tilde{L}_{Z}^{*}$, where $\tilde{L}_{Z}^{*}$ denote the conjugate operator of $\tilde{L}_{Z}$, let $\tilde{\varphi}_{1}^{*}$ denote the nonnegative eigenfunction of $\tilde{L}_{Z}^{*}$ corresponding to $\tilde{\lambda}_{1}(Z)$. Therefore, we have

$$
\tilde{\varphi}_{1}^{*}(t)=\tilde{\lambda}_{1}(Z) \tilde{L}_{Z}^{*} \tilde{\varphi}_{1}^{*}(t), \quad t \in[0,1] .
$$

Lemma 4.2 Let $\Lambda \subset \mathbb{R}^{+}$be a compact interval with $\left[\tilde{\lambda}_{1}\left(a^{0}\right), \tilde{\lambda}_{1}\left(a_{0}\right)\right] \cap \Lambda=\emptyset$. Then there exists a number $\delta_{1}>0$ such that

$$
\Phi_{\lambda}(u) \neq 0, \quad \forall \lambda \in \Lambda, \forall u \in P C[0,1]: 0<\|u\| \leq \delta_{1} .
$$

Proof Suppose on the contrary that there exists $\left\{\left(\mu_{n}, u_{n}\right)\right\} \subset \Lambda \times P C[0,1]$ with $\left\|u_{n}\right\| \rightarrow 0$ $(n \rightarrow \infty)$, such that $\Phi_{\mu_{n}}\left(u_{n}\right)=0$. We may assume $\mu_{n} \rightarrow \bar{\mu} \in \Lambda$. By Remark 2.2, $u_{n}>0$ in $[0,1]$. Set $v_{n}=\left\|u_{n}\right\|^{-1} u_{n}$. Then

$$
v_{n}=\frac{A_{\mu_{n}}\left(u_{n}\right)}{\left\|u_{n}\right\|} .
$$

From (H2), (H3), we know that $\left\|u_{n}\right\|^{-1} A_{\mu_{n}}\left(u_{n}\right)$ is bounded in $P C[0,1]$, so we infer that $v_{n}$ is a relatively compact set in $P C[0,1]$, hence (for a subsequence) $v_{n} \rightarrow \bar{v}$ with $\bar{v} \geq 0$ in $P C[0,1],\|\bar{v}\|=1$.

Now, from condition (H2), we know that there exist $\rho_{k}^{0} \in C([0, \infty),[0, \infty))$, such that

$$
I_{k}(u)=I_{k}^{(0)} \cdot u+\rho_{k}^{0}(u) \quad \text { and } \quad \lim _{u \rightarrow 0^{+}} \frac{\rho_{k}^{0}(u)}{u}=0 .
$$

From (H3), we have that

$$
a_{0}(t) u_{n}-\zeta_{1}\left(t, u_{n}\right) \leq f\left(t, u_{n}\right) \leq a^{0}(t) u_{n}+\zeta_{2}\left(t, u_{n}\right)
$$

So,

$$
u_{n} \leq \mu_{n} \int_{0}^{1} G(t, s) a^{0}(s) u_{n}(s) d s+\mu_{n} \sum_{k=1}^{p} G\left(t, t_{k}\right) I_{k}^{(0)} \cdot u_{n}\left(t_{k}\right)
$$




$$
+\mu_{n} \int_{0}^{1} G(t, s) \zeta_{2}\left(s, u_{n}(s)\right) d s+\mu_{n} \sum_{k=1}^{p} G\left(t, t_{k}\right) \rho_{k}^{0}\left(u_{n}\left(t_{k}\right)\right)
$$

and

$$
\begin{aligned}
u_{n} \geq & \mu_{n} \int_{0}^{1} G(t, s) a_{0}(s) u_{n}(s) d s+\mu_{n} \sum_{k=1}^{p} G\left(t, t_{k}\right) I_{k}^{(0)} \cdot u_{n}\left(t_{k}\right) \\
& -\mu_{n} \int_{0}^{1} G(t, s) \zeta_{1}\left(s, u_{n}(s)\right) d s+\mu_{n} \sum_{k=1}^{p} G\left(t, t_{k}\right) \rho_{k}^{0}\left(u_{n}\left(t_{k}\right)\right),
\end{aligned}
$$

accordingly, we have

$$
\begin{aligned}
v_{n} \leq & \mu_{n} \int_{0}^{1} G(t, s) a^{0}(s) v_{n}(s) d s+\mu_{n} \sum_{k=1}^{p} G\left(t, t_{k}\right) I_{k}^{(0)} \cdot v_{n}\left(t_{k}\right), \\
& +\mu_{n} \int_{0}^{1} G(t, s) \frac{\zeta_{2}\left(s, u_{n}(s)\right)}{u_{n}(s)} v_{n}(s) d s+\mu_{n} \sum_{k=1}^{p} G\left(t, t_{k}\right) \frac{\rho_{k}^{0}\left(u_{n}\left(t_{k}\right)\right)}{\left\|u_{n}\right\|}
\end{aligned}
$$

and

$$
\begin{aligned}
v_{n} \geq & \mu_{n} \int_{0}^{1} G(t, s) a_{0}(s) v_{n}(s) d s+\mu_{n} \sum_{k=1}^{p} G\left(t, t_{k}\right) I_{k}^{(0)} \cdot v_{n}\left(t_{k}\right) \\
& -\mu_{n} \int_{0}^{1} G(t, s) \frac{\zeta_{1}\left(s, u_{n}(s)\right)}{u_{n}(s)} v_{n}(s) d s+\mu_{n} \sum_{k=1}^{p} G\left(t, t_{k}\right) \frac{\rho_{k}^{0}\left(u_{n}\left(t_{k}\right)\right)}{\left\|u_{n}\right\|} .
\end{aligned}
$$

Let $\tilde{\varphi}_{*}^{0}$ and $\tilde{\varphi}_{0}^{*}$ denote the nonnegative eigenfunctions of $\tilde{L}_{a^{0}}^{*}, \tilde{L}_{a_{0}}^{*}$ corresponding to $\tilde{\lambda}_{1}\left(a^{0}\right)$, and $\tilde{\lambda}_{1}\left(a_{0}\right)$, respectively. Then we have from the (4.2) that

$$
\left\langle v_{n}, \tilde{\varphi}_{*}^{0}\right\rangle \leq \mu_{n}\left\langle\tilde{L}_{a^{0}} v_{n}, \tilde{\varphi}_{*}^{0}\right\rangle .
$$

Letting $n \rightarrow \infty$, we have

$$
\left\langle\bar{v}, \tilde{\varphi}_{*}^{0}\right\rangle \leq \bar{\mu}\left\langle\tilde{L}_{a^{0}} \bar{v}, \tilde{\varphi}_{*}^{0}\right\rangle,
$$

we obtain that

$$
\left\langle\bar{v}, \tilde{\varphi}_{*}^{0}\right\rangle \leq \bar{\mu}\left\langle\tilde{L}_{a^{0}} \bar{v}, \tilde{\varphi}_{*}^{0}\right\rangle=\bar{\mu}\left\langle\bar{v}, \tilde{L}_{a^{0}}^{*} \tilde{\varphi}_{*}^{0}\right\rangle=\bar{\mu}\left\langle\bar{v}, \frac{1}{\tilde{\lambda}_{1}\left(a^{0}\right)} \tilde{\varphi}_{*}^{0}\right\rangle=\bar{\mu} \frac{1}{\tilde{\lambda}_{1}\left(a^{0}\right)}\left\langle\bar{v}, \tilde{\varphi}_{*}^{0}\right\rangle,
$$

and consequently

$$
\bar{\mu} \geq \tilde{\lambda}_{1}\left(a^{0}\right)
$$

Similarly, we deduce from (4.3) that

$$
\bar{\mu} \leq \tilde{\lambda}_{1}\left(a_{0}\right) .
$$

Thus, $\tilde{\lambda}_{1}\left(a^{0}\right) \leq \bar{\mu} \leq \tilde{\lambda}_{1}\left(a_{0}\right)$. This contradicts $\bar{\mu} \in \Lambda$. 
Corollary 4.1 For $\mu \in\left(0, \tilde{\lambda}_{1}\left(a^{0}\right)\right)$ and $\delta \in\left(0, \delta_{1}\right)$. Then $\operatorname{deg}\left(\Phi_{\mu}, B_{\delta}, 0\right)=1$.

On the other hand, we have

Lemma 4.3 Suppose $\lambda>\tilde{\lambda}_{1}\left(a_{0}\right)$. Then there exists $\delta_{2}>0$ with the property that $\forall u \in$ $P C[0,1]$ with $0<\|u\| \leq \delta_{2}, \forall \tau \geq 0$,

$$
\Phi_{\lambda}(u) \neq \tau \tilde{\varphi}_{0}
$$

where $\tilde{\varphi}_{0}$ is the nonnegative eigenfunction of the $\tilde{L}_{a_{0}}$ corresponding to $\tilde{\lambda}_{1}\left(a_{0}\right)$.

Proof We assume again on the contrary that there exists $\tau_{n} \geq 0$ and a sequence $u_{n}$ with $\left\|u_{n}\right\|>0$ and $u_{n} \rightarrow 0$ in $P C[0,1]$, such that $\Phi_{\lambda}\left(u_{n}\right)=\tau_{n} \tilde{\varphi}_{0}$ for all $n \in \mathbb{N}$.

Then

$$
u_{n}=A_{\lambda}\left(u_{n}\right)+\tau_{n} \tilde{\varphi}_{0}
$$

and we conclude from Remark 2.2 that $u_{n}>0$ in $[0,1]$. So, we have

$$
\left\langle u_{n}, \tilde{\varphi}_{0}^{*}\right\rangle=\left\langle A_{\lambda}\left(u_{n}\right)+\tau_{n} \tilde{\varphi}_{0}, \tilde{\varphi}_{0}^{*}\right\rangle=\left\langle A_{\lambda}\left(u_{n}\right), \tilde{\varphi}_{0}^{*}\right\rangle+\tau_{n}\left\langle\tilde{\varphi}_{0}, \tilde{\varphi}_{0}^{*}\right\rangle .
$$

Choose $\sigma>0$ such that

$$
\sigma<\frac{\lambda-\tilde{\lambda}_{1}\left(a_{0}\right)}{\lambda} .
$$

By $(\mathrm{H} 3)$, there exists $r>0$, such that

$$
f(t, u) \geq(1-\sigma) a_{0}(t) u, \quad \forall u \in[0, r], t \in[0,1] .
$$

From $\left\|u_{n}\right\| \rightarrow 0$, then exists $N^{*}>0$, such that

$$
0 \leq u_{n} \leq r, \quad \forall n \geq N^{*}
$$

and consequently

$$
f\left(t, u_{n}\right) \geq(1-\sigma) a_{0}(t) u_{n}, \quad \forall n \geq N^{*} .
$$

Let $v_{n}=\frac{u_{n}}{\|u\|}$, applying (4.5), it follows that

$$
\begin{aligned}
\left\langle v_{n}, \tilde{\varphi}_{0}^{*}\right\rangle & \geq\left\langle\frac{A_{\lambda}\left(u_{n}\right)}{\left\|u_{n}\right\|}, \tilde{\varphi}_{0}^{*}\right\rangle \geq \lambda(1-\sigma)\left\langle\tilde{L}_{a_{0}} v_{n}, \tilde{\varphi}_{0}^{*}\right\rangle \\
& =\lambda(1-\sigma)\left\langle v_{n}, \tilde{L}_{a_{0}}^{*} \tilde{\varphi}_{0}^{*}\right\rangle=\lambda(1-\sigma)\left\langle v_{n}, \frac{1}{\tilde{\lambda}_{1}\left(a_{0}\right)} \tilde{\varphi}_{0}^{*}\right\rangle .
\end{aligned}
$$

Thus,

$$
\tilde{\lambda}_{1}\left(a_{0}\right) \geq \lambda(1-\sigma)
$$

this contradicts with (4.4). 
Corollary 4.2 For $\lambda>\tilde{\lambda}_{1}\left(a_{0}\right)$ and $\delta \in\left(0, \delta_{2}\right)$. Then $\operatorname{deg}\left(\Phi_{\lambda}, B_{\delta}, 0\right)=0$.

Proof By Lemma 4.3, there exists $\delta_{2}>0$ such that

$$
\Phi_{\lambda}(u) \neq \tau \tilde{\varphi}_{0}, \quad \forall u \in P C[0,1]: 0<\|u\| \leq \delta_{2}, \tau \in[0,1] .
$$

Then

$$
\operatorname{deg}\left(\Phi_{\lambda}, B_{\delta}, 0\right)=\operatorname{deg}\left(\Phi_{\lambda}-\tilde{\varphi}_{0}, B_{\delta}, 0\right)=0
$$

for all $\delta \in\left(0, \delta_{2}\right)$. Then the assertion follows.

Now, using Theorem $\mathrm{C}$ and the similar method to prove Proposition 3.1 with obvious changes, we may prove the following proposition.

Proposition $4.1\left[\tilde{\lambda}_{1}\left(a^{0}\right), \tilde{\lambda}_{1}\left(a_{0}\right)\right]$ is a bifurcation interval of positive solutions from the trivial solution for the problem (2.4). There exists an unbounded component $\Sigma_{0}$ of positive solutions of (2.4) which meets $\left[\tilde{\lambda}_{1}\left(a^{0}\right), \tilde{\lambda}_{1}\left(a_{0}\right)\right] \times\{0\}$. Moreover, there exists no bifurcation interval of positive solutions from the trivial solution which is disjointed with $\left[\tilde{\lambda}_{1}\left(a^{0}\right), \tilde{\lambda}_{1}\left(a_{0}\right)\right]$.

This is exactly the assertion (ii) of Theorem 1.1.

\section{Global behavior of the component of positive solutions}

In this section, we consider the intertwining of the branches bifurcating from infinity and from the trivial solution.

Let $m_{k}:=\min \left\{\frac{I_{k}(u)}{u}\right\}, k=1, \ldots, p$ for $u \neq 0$. From $(\mathrm{H} 2)$, we have $m_{k}>0, k=1, \ldots, p$.

Define the linear operator $T_{c}: P C[0,1] \rightarrow P C[0,1]$,

$$
T_{c} u=\int_{0}^{1} G(t, s) c(s) u(s) d s+\sum_{k=1}^{p} G\left(t, t_{k}\right) m_{k} \cdot u\left(t_{k}\right)
$$

where $c(\cdot)$ is defined in (H5), $G(t, s)$ is defined in (2.2).

Similar as Lemma 2.2, we have the following lemma.

Lemma 5.1 The operator $T_{c}$ has a unique characteristic value $\mu_{1}$, which is positive, real, simple, and the corresponding eigenfunction $\Phi_{c}(t)$ is of one sign, i.e., we have $\Phi_{c}(t)=$ $\mu_{1} T_{c} \Phi_{c}(t)$.

Remark 5.1 Since $\mu_{1}$ is real number, so from Lemma 2.1, $\mu_{1}$ is also the characteristic value of $T_{c}^{*}$, where $T_{c}^{*}$ denote the conjugate operator of $T_{c}$, let $\Phi_{c}^{*}$ denote the nonnegative eigenfunction of $T_{c}^{*}$ corresponding to $\mu_{1}$. Therefore, we have

$$
\Phi_{c}^{*}(t)=\mu_{1} T_{c}^{*} \Phi_{c}^{*}(t), \quad t \in[0,1] .
$$

Lemma 5.2 Let (H1)-(H5) hold. Then there exists a number $\lambda^{*}>0$ such that there is no positive solution $(\lambda, u)$ of $\Phi_{\lambda}(u)=0$ with $\lambda>\lambda^{*}$. 
Proof Let $(\lambda, u)$ be a positive solution of $\Phi_{\lambda}(u)=0$. Then

$$
u=\lambda \int_{0}^{1} G(t, s) f(s, u(s)) d s+\lambda \sum_{k=1}^{p} I_{k}\left(u\left(t_{k}\right)\right), \quad u \in P C[0,1]
$$

From (H5) and the definition of $m_{k}$, we have

$$
u \geq \lambda \int_{0}^{1} G(t, s) c(s) u(s) d s+\lambda \sum_{k=1}^{p} m_{k} \cdot u\left(t_{k}\right), \quad u \in P C[0,1] .
$$

From (5.2), we have

$$
\left\langle u, \Phi_{c}^{*}\right\rangle \geq \lambda\left\langle T_{c} u, \Phi_{c}^{*}\right\rangle=\lambda\left\langle u, T_{c}^{*} \Phi_{c}^{*}\right\rangle=\lambda\left\langle u, \frac{1}{\mu_{1}} \Phi_{c}^{*}\right\rangle=\lambda \frac{1}{\mu_{1}}\left\langle u, \Phi_{c}^{*}\right\rangle .
$$

Thus,

$$
\lambda \leq \mu_{1}:=\lambda^{*} .
$$

The assertion that $\Sigma_{0}=\Sigma_{\infty}$ in Theorem 1.1(iii) now easily follows. For, in the case, $\Sigma_{0}$ and $\Sigma_{\infty}$ are contained in $\left(0, \lambda^{*}\right] \times P C[0,1]$. Moreover, there exists no bifurcation interval of positive solution from infinity which is disjointed with $\left[\lambda_{1}\left(b^{\infty}\right), \lambda_{1}\left(b_{\infty}\right)\right]$, there exists no bifurcation interval of positive solution from the trivial solution which is disjointed with $\left[\tilde{\lambda}_{1}\left(a^{0}\right), \tilde{\lambda}_{1}\left(a_{0}\right)\right]$. In Theorem 1.1(iii), the unbounded component $\Sigma_{0}$ has to meet $\left[\lambda_{1}\left(b^{\infty}\right), \lambda_{1}\left(b_{\infty}\right)\right] \times\{\infty\}$.

\section{Competing interests}

The authors declare that they have no competing interests.

\section{Authors' contributions}

RM completed the main study and carried out the results of this article. BY drafted the manuscript. ZW checked the proofs and verified the calculation. All the authors read and approved the final manuscript.

\section{Acknowledgements}

The authors are very grateful to the anonymous referees for their valuable suggestions. This work was supported by the NSFC (No. 11061030), NSFC (No. 11126296), and the Fundamental Research Funds for the Gansu Universities.

Received: 18 May 2012 Accepted: 20 July 2012 Published: 1 August 2012

\section{References}

1. Bainov, DD, Simeonov, PS: Impulsive Differential Equations: Periodic Solutions and Applications. Longman, Harlow (1993)

2. He, ZM, Yu, JS: Periodic boundary value problem for first-order impulsive ordinary differential equations. J. Math. Anal. Appl. 272, 67-78 (2002)

3. Nieto, JJ: Periodic boundary value problems for first-order impulsive ordinary differential equations. Nonlinear Anal. TMA 51, 1223-1232 (2002)

4. Li, JL, Nieto, JJ, Shen, JH: Impulsive periodic boundary value problems of first-order differential equations. J. Math. Anal. Appl. 325, 226-236 (2007)

5. Zhao, AM, Bai, ZG: Existence of solutions to first-order impulsive periodic boundary value problems. Nonlinear Anal. TMA 71, 1970-1977 (2009)

6. Zhang, XY, Li, XY, Jiang, DQ, Wang, K: Multiplicity positive solutions to periodic problems for first-order impulsive differential equations. Comput. Math. Appl. 52, 953-966 (2006)

7. Liu, YJ: Positive solutions of periodic boundary value problems for nonlinear first-order impulsive differential equations. Nonlinear Anal. TMA 70, 2106-2122 (2009)

8. Chu, JF, Nieto, J: Impulsive periodic solutions of first-order singular differential equations. Bull. Lond. Math. Soc. 40 143-150 (2008)

9. Liu, Y, O'Regan, D: Multiplicity results using bifurcation techniques for a class of boundary value problems of impulsive differential equations. Commun. Nonlinear Sci. Numer. Simul. 16, 1769-1775 (2011) 
10. $\mathrm{Ma}, \mathrm{RY}, \mathrm{Xu}, \mathrm{J}$ : Bifurcation from interval and positive solutions of a nonlinear fourth-order boundary value problem. Nonlinear Anal. TMA 72, 113-122 (2010)

11. $\mathrm{Xu}, \mathrm{J}, \mathrm{Ma}, \mathrm{RY}$ : Bifurcation from interval and positive solutions for second order periodic boundary value problems. Appl. Math. Comput. 216, 2463-2471 (2010)

12. Schmitt, K, Thompson, RC: Nonlinear Analysis and Differential Equations: An Introduction, University of Utah Lecture Note. University of Utah, Salt Lake City (2004)

13. Schmitt, K: Positive Solutions of Semilinear Elliptic Boundary Value Problem, pp. 447-500. Kluwer Academic, Dordrecht (1995)

14. Kato, T: Perturbation Theory for Linear Operators. Springer, New York (1980)

15. Deimling, K: Nonlinear Functional Analysis. Springer, Berlin (1985)

doi:10.1186/1687-2770-2012-83

Cite this article as: Ma et al.: Bifurcation of positive periodic solutions of first-order impulsive differential equations. Boundary Value Problems 2012 2012:83.

Submit your manuscript to a SpringerOpen ${ }^{\odot}$ journal and benefit from:

- Convenient online submission

- Rigorous peer review

- Immediate publication on acceptance

- Open access: articles freely available online

- High visibility within the field

- Retaining the copyright to your article

Submit your next manuscript at $>$ springeropen.com 